\title{
Wearable self-tuning antenna for emergency rescue operations
}

\author{
Andrea Baroni ${ }^{1} \bowtie$, Paolo Nepa ${ }^{1}$, Hendrik Rogier ${ }^{2}$ \\ ${ }^{1}$ Department of Information Engineering, University of Pisa, Via G. Caruso 16, Pisa, Italy \\ ${ }^{2}$ Department of Information Technology, Ghent University, Sint-Pietersnieuwstraat 41, Ghent, Belgium \\ $凶$ E-mail: andrea.baroni@for.unipi.it
}

\begin{abstract}
In this study, the design, hardware implementation and characterisation of a self-tuning $406 \mathrm{MHz}$ antenna of a Cospas-Sarsat personal locator beacon are presented. The realised prototype is able to perform automatic tuning of the antenna under time-varying environmental conditions due to human body movements and sea water proximity. The impedance tuning is performed by tracking the instantaneous value of the reflection coefficient and by modifying an appropriate impedance matching network according to a real-time adaptive algorithm. A resilient default/backup software architecture has been designed to ensure that tuning guarantees a return loss higher than $10 \mathrm{~dB}$ for the personal locator beacon in most of impedance mismatching conditions.
\end{abstract}

\section{Introduction}

The International Cospas-Sarsat Programme [1] is a satellite-based search and rescue (SAR) distress alert detection and information distribution system, best known for detecting and locating emergency beacons activated by aircraft, ships and backcountry hikers in distress. The International Cospas-Sarsat Programme provides accurate, timely, and reliable distress alert and location data to help search and rescue authorities assist persons in distress. During an emergency situation, a Cospas-Sarsat radio-beacon transmits a distress signal to a constellation of satellites (LEOSAR and GEOSAR). Specifically, a $406 \mathrm{MHz}$ personal locator beacon emits a $5 \mathrm{~W}$ half-second burst approximately every $50 \mathrm{~s}$ [2]. The carrier frequency is phase-modulated with a digital message. This emergency message is then forwarded to a Ground Receiving Station, which informs a Rescue Coordinator Centre for the final rescue operations.

The integration of such a system directly inside a commercial life-jacket increases the chances of survival of a human in an emergency scenario. However, when a personal locator beacon with a radiating element is located near to a human body, re-orientation and movements of the wearer may continuously modify the operating conditions of the transmit antenna due to random variations of the relative position of the antenna with respect to the human body [3-5]. Moreover, in a maritime scenario, the presence of the sea water may affect the principal radiating parameters (input impedance and radiation pattern) of a wearable antenna [6]. Detuning of the antenna may cause a serious impedance mismatch and load pulling in the power amplifier (PA), resulting in a significant portion of the $5 \mathrm{~W}$ transmitter output signal being reflected back to the generator. Besides a serious reduction of the system efficiency, this reflected power may damage the source circuit. Special measures may be taken to protect the generator, such as the use of circulators, but this increases size and cost of the system and it reduces the power efficiency and, hence, the autonomy of the search and rescue system.

In this paper, we propose a novel self-tuning Cospas-Sarsat antenna that adaptively and in real-time compensates potential detuning to maintain the reflection coefficient below the $-14 \mathrm{~dB}$ threshold, while still guaranteeing an antenna gain between -3 and $4 \mathrm{dBi}$ over $90 \%$ of the region defined by all azimuth angles and by elevation angles greater than $5^{\circ}$ and less than $60^{\circ}$, as required by the Cospas-Sarsat specification document [7]. The self-tuning network automatically reconfigures itself, by tracking the antenna impedance variations caused by the movements of the wearer. The proposed solution estimates the mismatch level by measuring both the absolute value and the phase of the actual reflection coefficient. Continuous impedance matching regulation is performed by using a simple circuit based on varicap diodes. Robust minimisation of impedance mismatch is performed by a resilient default/backup software architecture solution. In normal operating conditions, an adaptive algorithm performs impedance matching based on both absolute value and phase of the reflection coefficient. However, when one of these quantities becomes unavailable, the microcontroller still ensures impedance matching by switching to one of two different backup algorithms that minimise the impedance mismatch based on one parameter only. Such a failsafe implementation improves the overall reliability of the system in an emergency situation. The self-tuning system guarantees that vital information is received by the Cospas-Sarsat satellites during a rescue operation, by maintaining antenna matching, gain and autonomy during the required complete $24 \mathrm{~h}$ timeframe. The alternative solution, which consists of deploying an isolator before the antenna, to protect the PA by absorbing the reflected power, will reduce the system autonomy and the antenna gain. As an example, a worst-case reflection coefficient of $-4.1 \mathrm{~dB}$, as observed in the experiments performed in Section 2, would require that almost $2 \mathrm{~W}$ of power (being $39 \%$ of the power delivered by the PA) is absorbed by the isolator load, reducing the antenna gain by $2.1 \mathrm{~dB}$ and limiting the system autonomy to $15 \mathrm{~h}$ and $16 \mathrm{~min}$ instead of $24 \mathrm{~h}$, if the same battery is applied.

On the one hand, different kinds of wearable antennas integrated on commercial life-jackets have been presented over the past years for the Cospas-Sarsat system at the ultra-high frequency of 406 $\mathrm{MHz}[6,8]$. However, these designs do not fully consider the effect of variations in the antenna's operating conditions due to the wearer re-orientation and movement. On the other hand, closed-loop self-tuning impedance networks were investigated for other applications $[9,10]$. In the open literature, different designs of self-tuning circuits are reported. A first characterisation aspect is related to the collection of the feedback data. One potential solution consists in measuring current or voltage values at different points of the circuit $[11,12]$. An alternative possibility is to sense the signals before and after the impedance matching network to determine the impedance mismatching level [13]. Other designs rely on directional couplers to monitor the direct and 


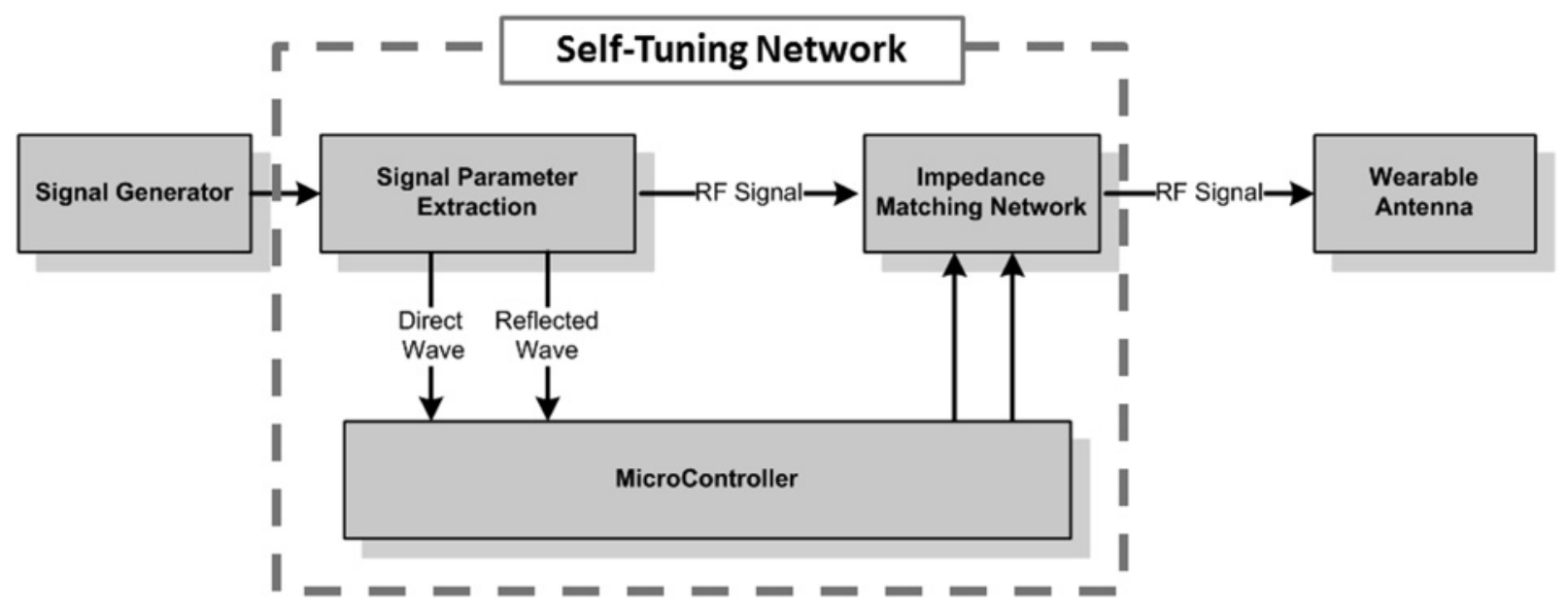

Fig. 1 Closed-loop self-tuning network concept

reflected waves [14-16], from which the reflection coefficient may be calculated. The adaptive algorithm that modifies the impedance matching network must be customised to the type of impedance matching circuit deployed. Some papers $[17,18]$ propose a discrete impedance matching network, consisting of a bank of capacitors or inductors, together with global optimisation algorithms such as the genetic algorithm, simulated annealing or the ant colony optimisation [19]. However, this setup occupies a lot of space, which may not be available on a commercial life-jacket. To miniaturise the electronic circuit, microelectromechanical systems (MEMS) technology is applied in [20, 21]. Nevertheless, this technology does not result in a low cost solution and its use in stub-based or transmission-line-based MEMS matching networks is impractical at the Cospas-Sarsat frequencies, as even miniaturisation by the use of slow-wave structures leads matching circuit footprints in the order of the wavelength. In other applications, the use of varicap diodes [2224] has resulted in low cost and compact reconfigurable solutions. For tunable capacitors, a lookup table may be used to predict the optimal capacitor value [25]. However, such an approach does not take into account the tolerance on every component in the matching network. Therefore, in this paper, a dedicated software architecture has been developed.

The outline of the paper is organised as follows. In Section 2, a brief concept of the system and an experimental prototype are reported. The implemented tuning control algorithms are described in Section 3. Measured results are discussed in Section 4. Finally, in Section 5, conclusions are drawn.

\section{Self-tuning network}

The conventional structure of a self-tuning network is depicted in Fig. 1. The closed-loop system tracks the instantaneous value of the reflection coefficient to modify a number of parameters in the impedance matching network.

The measurement of the reflection coefficient is performed by two three-port directional couplers and a gain/phase detector. The directional couplers sense the direct and reflected waves in the transmit chain. The gain/phase detector receives these waves as inputs and returns two analogue voltage values proportional to the ratio and the phase difference between the two input signals (being the absolute value and the phase of the reflection coefficient). Using the algorithms explained in Section 3, the microcontroller then interprets these data to achieve impedance matching.

The impedance matching network is the last component of the self-tuning circuit. To fix its topology and circuit component values, first the potential variations in the reflection coefficient were quantified by measuring the reflection coefficients of a meandered dipole Cospas-Sarsat antenna [6], when worn by a volunteer performing random movements and when floating on salt water (salinity level of $35 \%$ ) in a recipient that was slightly moved in a continuous manner, to emulate variation of the sea surface, caused, for example, by the sea waves. The measurement results are graphically shown in Fig. $2 a$. To obtain a better view on the design challenge, Table 1 presents some worst-case results for the different scenarios. When the antenna is deployed on the water, the reflection coefficient can assume values up to $-4.1 \mathrm{~dB}$. In this case, $39 \%$ of the total output power is lost due to reflections, whereas only $61 \%$ is available for antenna radiation. If we do not compensate for this mismatch, a power of several watts will have to be dissipated somewhere in the transmit chain (e.g. by a bulky and costly drop-in isolator), reducing the antenna total gain and the system autonomy. Therefore, we prefer to compensate for the mismatch by proposing the tuning network topology depicted in Fig. $2 b$.

To implement the final circuit, first the tuning elements are selected. The MMBV105GLT1G [26] varicap diodes exhibit a junction capacitance ranging from 4 to $18 \mathrm{pF}$, when a reverse voltage of 12 and $0.5 \mathrm{~V}$ is applied, respectively. Fig. $2 c$ shows the tuning range of this network with these components, tuned over the ranges described in Table 2. To cover the complete capacitance range put forward in Table 1 with the varicap diodes, some modifications of the circuit topology in Fig. $2 b$ are needed. The variable range of the capacitor $C_{1}$ equals $7-20 \mathrm{pF}$. This range may be covered by a series connection of a single varicap diode and a single shunt $2 \mathrm{pF}$ bias capacitor, shifting the dynamic available range to $6-20 \mathrm{pF}$. As for $C_{2}$, to cover the simulated dynamic range of $30 \mathrm{pF}$, three varicap diodes are deployed together with a shunt inductor of $15 \mathrm{nH}$, to shift the dynamic capacitance range from $12-54$ to $2-44 \mathrm{pF}$. Finally, a set of choke inductors and DC blocks are added to block the radio-frequency (RF) signal towards the microcontroller and to isolate the DC commands sent to different varicap diodes, respectively. Above considerations result in the impedance matching circuit shown in Fig. 3a. An external digital-to-analogue converter has also been added to control the analogue reverse voltage applied to the varicap diodes through the digital output pins of the microcontroller. Adopting a different microcontroller with integrated analogue outputs could avoid the need for such a digital-to-analogue converter. The final structure of the self-tuning network is depicted in Fig. $3 b$. It is the result of a comprehensive analysis procedure where the best network topology and components were selected in terms of small dimensions and low cost, to keep the overall size and the price as small as possible. Therefore, in the impedance matching network, only passive surface-mount device (SMD) components with a unit cost lower than $0.5 €$ have been deployed. The selected varicap diodes cost $0.22 €$ per piece. Special attention should be devoted to the directional coupler. Many suitable off-the-shelf-hybrid couplers, such as the MACP-011013 [27], are available at a price in the 

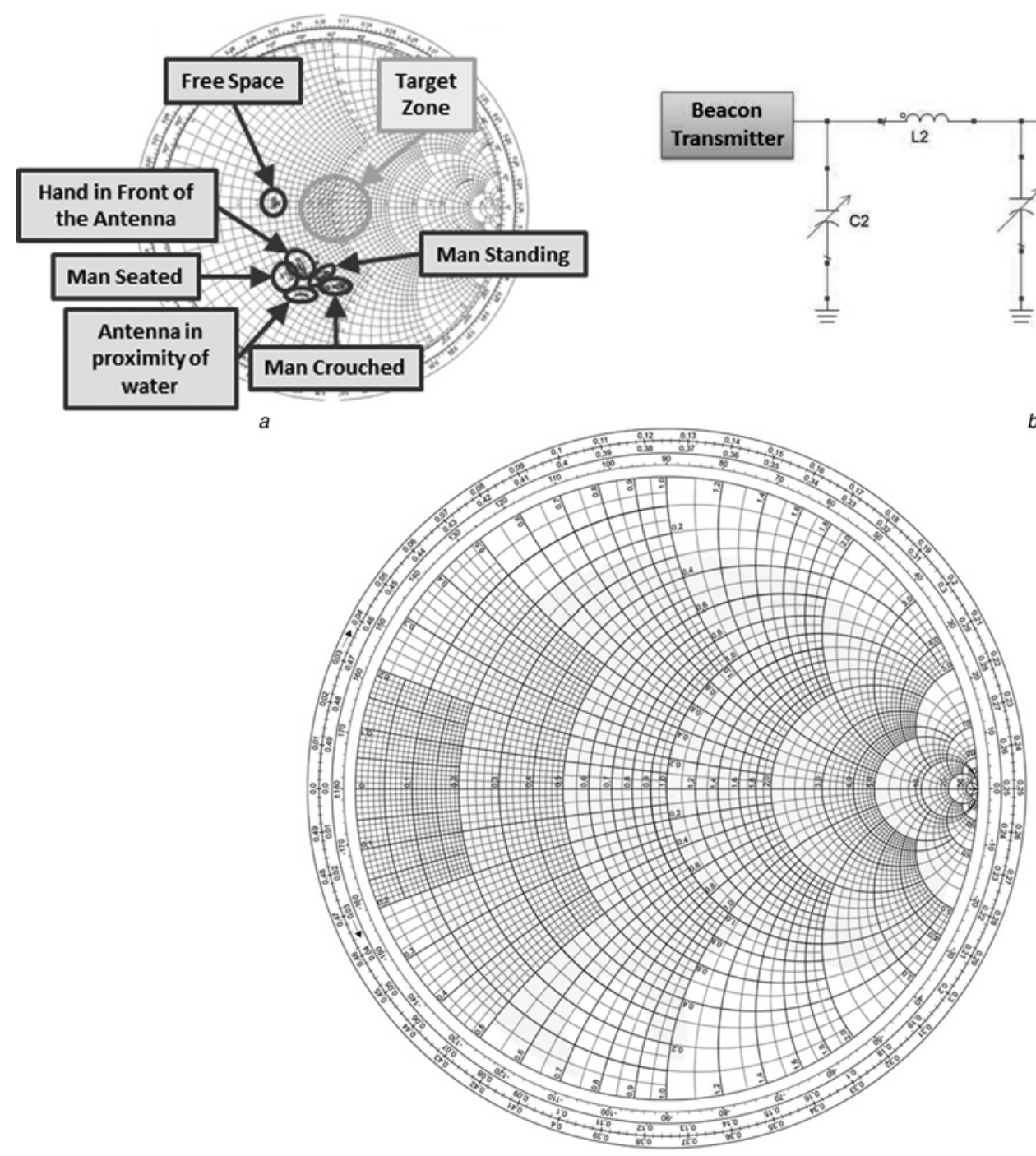

Fig. 2 Reflection coefficient and impedance network

$a$ Measured reflection coefficient on the Smith chart for different movements of the wearer

$b$ Identified double low-pass-upward- $L$ tuning network

$c$ Simulated matching domain by the realized network

order of $1 €$ per component. Moreover, in the final integration, the microcontroller and the gain/phase detector used for the antenna section can be shared with the Cospas-Sarsat transmitter to reduce the price even further. All these considerations limit the cost of the impedance matching network to a small fraction of the overall module, taking into account that a commercial life-jacket has a price of around $20 \$$ and the average cost of a commercial Cospas-Sarsat transmitter is around $200 \$$.

The integration of the self-tuning network into the floatation device of a commercial life-jacket is shown in Fig. 3c. The antenna is located on the upper side of the floating element,

Table 1 Worst-case reflection coefficient, antenna total gain reduction and power reflected due to antenna mismatch, for different antenna operating conditions, assuming $5 \mathrm{~W}$ injected power

\begin{tabular}{lccc}
\hline $\begin{array}{l}\text { Position of the } \\
\text { antenna }\end{array}$ & $\begin{array}{c}\text { Reflection } \\
\text { coefficient, dB }\end{array}$ & $\begin{array}{c}\text { Total gain } \\
\text { reduction, dB }\end{array}$ & $\begin{array}{c}\text { Max reflected } \\
\text { power, W (\%) }\end{array}$ \\
\hline $\begin{array}{l}\text { free space } \\
\text { hand in front of } \\
\text { the antenna }\end{array}$ & -8.7 & 0.63 & $0.674(13)$ \\
man standing & -6.1 & 1.22 & $1.227(24)$ \\
man seated & -6.8 & 1.02 & $1.045(21)$ \\
man crouched & -5.7 & 1.36 & $1.346(27)$ \\
water proximity & -6.0 & 1.26 & $1.256(25)$ \\
\hline
\end{tabular}

whereas the self-tuning network is integrated inside the floating element. Tests have been conducted to verify the behaviour of the self-tuning network when deployed near the radiating element. These tests have demonstrated that the proximity of the antenna does not affect the impedance matching procedure.

\section{Tuning algorithms}

The tuning algorithms form the functional core of the system, as they act on the impedance matching network to minimise the impedance mismatch of the narrowband Cospas-Sarsat antenna. Therefore, they rely on the incident and reflected waves between antenna and tuning element, which are monitored in real time. The control unit iterates until the best matching solution is achieved along a tuning

Table 2 Value of the tuning circuit components

\begin{tabular}{lc}
\hline Component name & Component value \\
\hline$L_{1}$ & $29 \mathrm{nH}$ \\
$C_{1}$ & from 7 to $20 \mathrm{pF}$ \\
$L_{2}$ & $19.6 \mathrm{nH}$ \\
$C_{2}$ & from 2 to $30 \mathrm{pF}$ \\
\hline
\end{tabular}



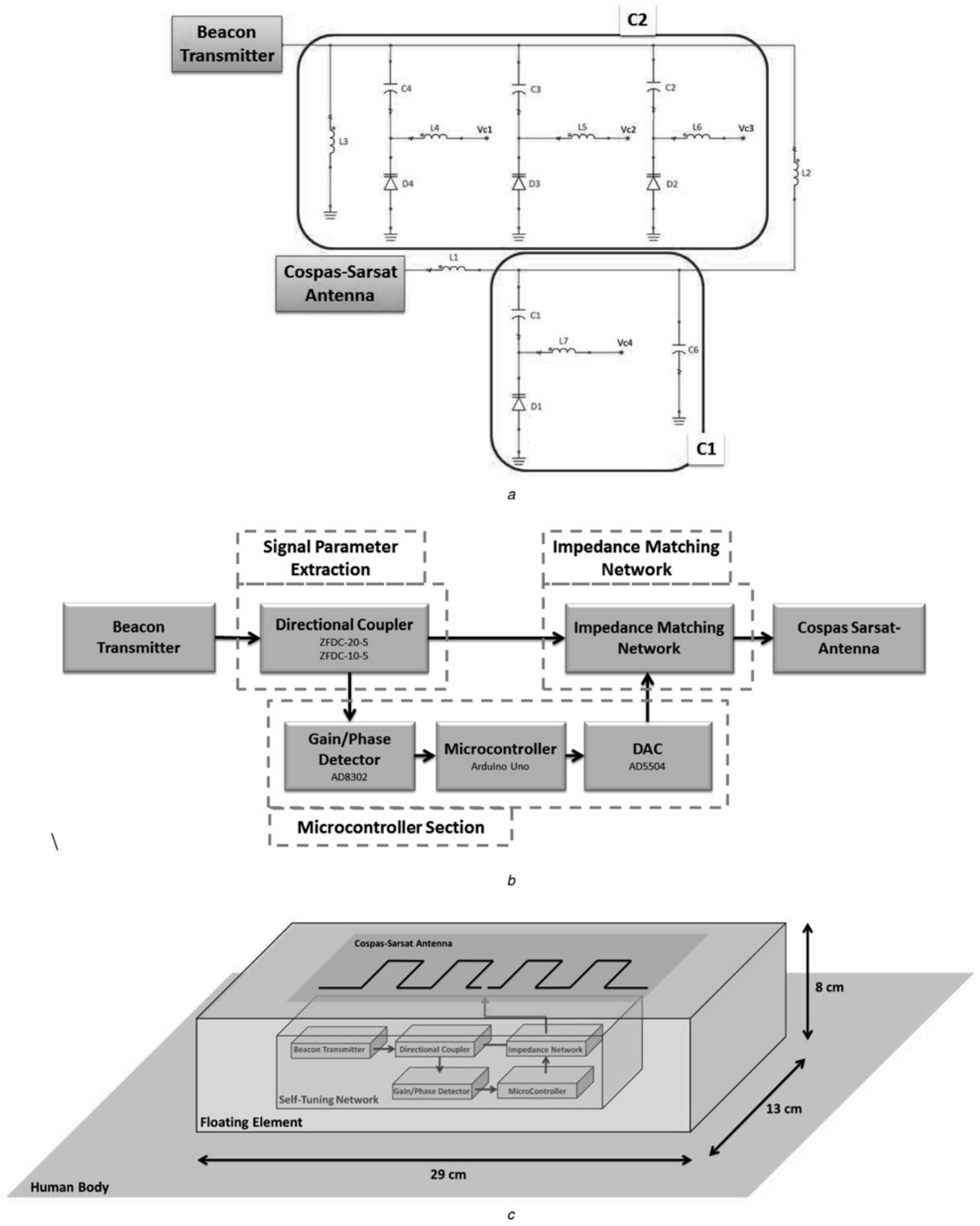

Fig. 3 Integration process of the self-tuning antenna, from the impedance network to the integration into the floatation device $a$ Schematic design of the identified impedance network $b$ Final circuit for the self-tuning impedance network

$c$ Schematic overview of the antenna and self-tuning network integrated inside the floatation device

trajectory controlled by the optimisation algorithm, starting from an arbitrary point in the two-dimensional impedance plane.

A look-up table mechanism could be applied to calculate the exact values of the varicap diodes. However, this method has been discarded, mainly due to component tolerances and temperaturedependent variations in the physical components employed in the impedance matching network, which may affect the correctness of impedance matching based on fixed lookup table values. Hence, optimal impedance matching cannot be achieved by a static approach. Therefore, a dynamic adaptation strategy was adopted.

A robust failsafe software architecture that relies on three different kinds of algorithms has been implemented (Fig. 4a). All of them 


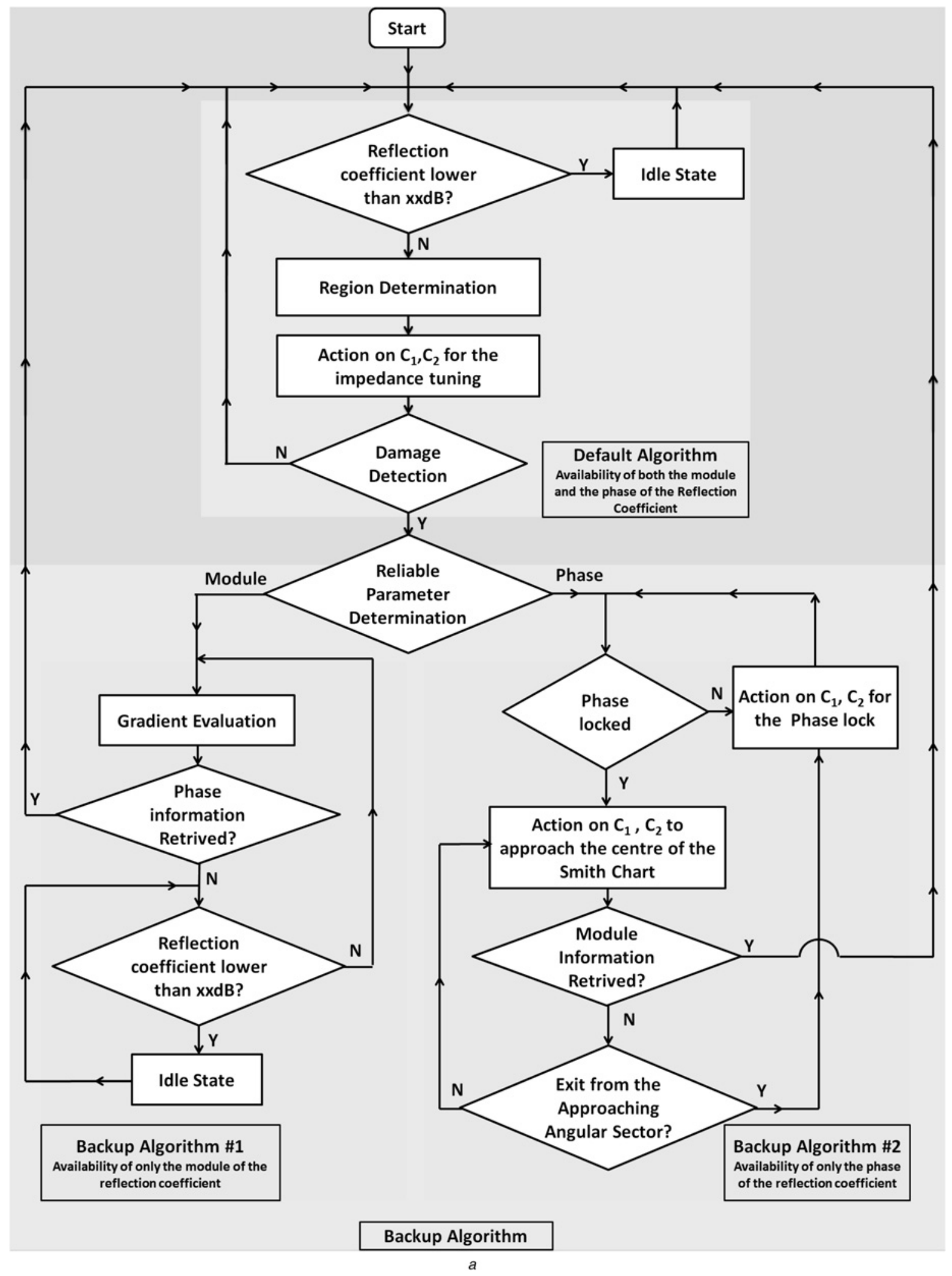

Fig. 4 Software architecture implemented on the microcontroller of the self-tuning antenna

$a$ Flowchart of the software architecture

$b$ Description of the default algorithm

$c$ Description of the amplitude-based backup algorithm

$d$ Description of the phase-based backup algorithm

were programmed on the microcontroller and tested. A default algorithm achieves impedance matching based on all the physical quantities that can be provided by the gain/phase detector, being both the absolute value and the phase of the reflection coefficient (Fig. $4 b$ ). In addition, two backup algorithms are available in case of some malfunction of either the gain or phase detector, or when deploying off-the-shelf components able to provide only one of the two mentioned quantities to the microcontroller (Figs. $4 b$ and $c)$. In that case, they perform the impedance matching by relying on only one physical quantity measured by the gain/phase 


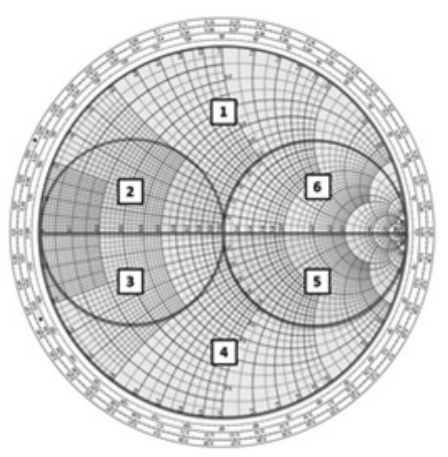

\section{Default algorithm}

Input data: Reflection coefficient amplitude and phase.

Summary: The Smith Chart is subdivided into 6 regions.

According to the specific location of the reflection coefficient, the variable components in the impedance matching network are adapted as described in Table 2.

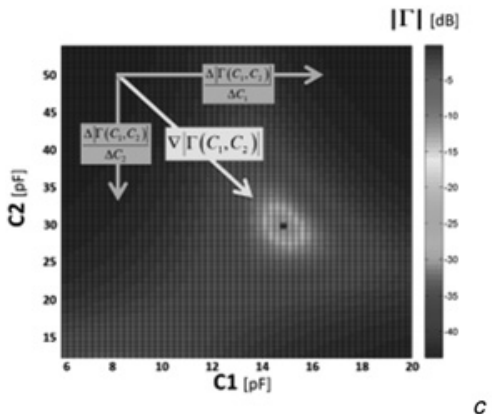

\section{Backup Algorithm \#1}

Input data: Reflection coefficient amplitude.

Summary: The minimization of the reflection coefficient is performed based on its local gradient in the $\left(\mathrm{C}_{1}, \mathrm{C}_{2}\right)$ plane.

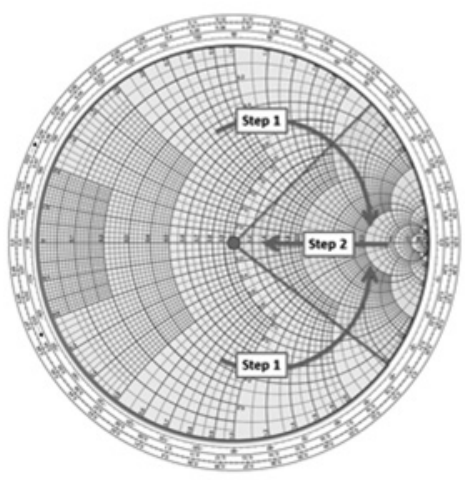

Backup Algorithm \#2

Input data: Reflection coefficient phase

Summary: The minimization process proceeds in two different phases. In the first step, the reflection coefficient value is led into the phase-lock region, being an angular region on the Smith Chart. In the subsequent step, the reflection coefficient is reduced towards the centre of the Smith Chart. The definition of an angular region is needed to be able to control the proximity of the reflection to the centre of the Smith Chart, given that amplitude information is unavailable.

Fig. 4 Continued

detector, being either the amplitude or the phase of the reflection coefficient. Hence, when the microcontroller detects that either the amplitude or the phase measurement is unavailable, or its data are unreliable, it switches to the most appropriate algorithm to still ensure impedance matching.

In the default algorithm, the entire Smith chart is subdivided into six different regions, based on the real (larger or smaller than 1) and imaginary parts (positive or negative) of the normalised impedance and on the real part of the admittance (greater or lower than 1). The knowledge of both the amplitude and the phase of the reflection coefficient permits to identify the region in which the reflection coefficient is located.

For every region, the actions performed on the two variable elements are implemented based on considerations about the tuning circuit shown in Fig. $2 b$. On the one hand, shunt capacitor $C_{2}$ only modifies the load susceptance; therefore, it only allows movements along conductance circles on the Smith chart. On the other hand, shunt capacitor $C_{1}$ affects both real and imaginary parts of the reflection coefficient. Therefore, with this component we may select a different conductance circle (Fig. 5).

On the basis of the above considerations, capacitor $C_{1}$ is tuned to compensate for the real part of the impedance (by reaching the admittance unit circle or the impedance unit circle), while

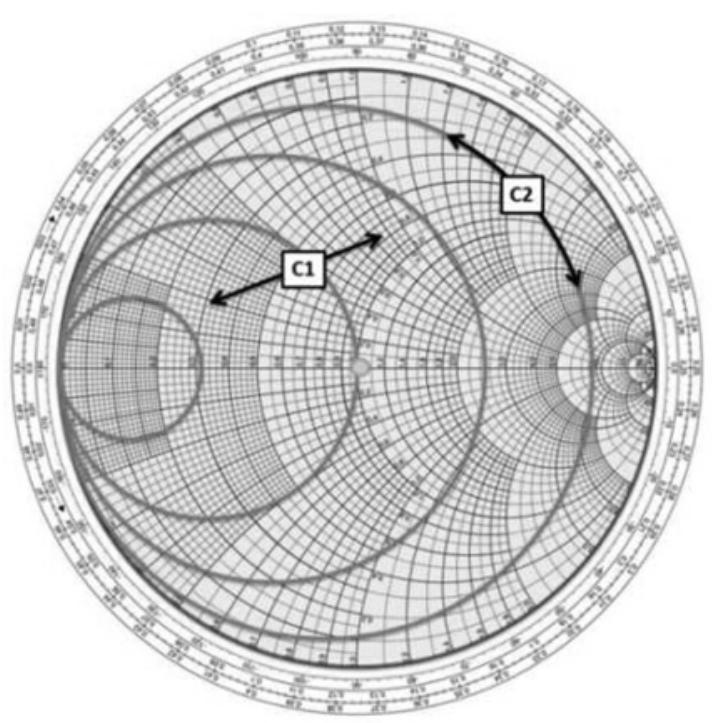

Fig. 5 Effect of the variations of the two variable capacitors 
Table 3 Action on the two variable capacitors for the default algorithm

\begin{tabular}{lll}
\hline Region & $C_{1}$ action & $C_{2}$ action \\
\hline 1 & decrease capacitance & decrease capacitance \\
2 & increase capacitance & decrease capacitance \\
3 & increase capacitance & increase capacitance \\
4 & decrease capacitance & increase capacitance \\
5 & decrease capacitance & increase capacitance \\
6 & decrease capacitance & decrease capacitance \\
\hline
\end{tabular}

capacitor $C_{2}$ compensate for the load susceptance. Adaptations performed on the two variable capacitors depend on the particular region on the Smith chart in which the reflection coefficient is located. Real-time tuning is achieved by modifying the variable components according to the actions described in Table 3 .

This process (determining the correct region and adapting the variable components) is repeated until optimal matching is reached. The availability of the magnitude of the real-time reflection coefficient as a feedback signal also permits to define a stopping criterion. For instance, we may aim for a magnitude of the reflection coefficient that is lower than $-15 \mathrm{~dB}$. Once the magnitude threshold is reached, the microcontroller can be set to standby mode, decreasing its energy consumption and enlarging the system autonomy.
The first backup algorithm only relies on the measured magnitude of the reflection coefficient to achieve impedance matching. The absolute value of the reflection coefficient depends on both the impedance of the antenna and the impedance matching circuit. On the one hand, variations on the antenna impedance matching cannot be controlled, as they depend on the working environment and on the wearer movements. On the other hand, the state of the tuning network can be controlled by the microcontroller. Therefore, the minimisation is performed by exploiting its local gradient with respect to the two variable elements $C_{1}$ and $C_{2}$. The estimation of the local gradient is performed by measuring the partial derivative components along the single dimension (both $C_{1}$ and $C_{2}$ ). Also in this case, the knowledge of the magnitude of the reflection coefficient is used to define a stopping criterion.

The second backup algorithm only relies on the feedback of the reflection coefficient's phase. As no knowledge is available about the quantity to be minimised, being the magnitude, special care should be taken when performing the impedance matching. The developed algorithm is based on a two-step process, which first modifies the reflection coefficient's phase, bringing it into a phase-lock region, after which reflection coefficient's amplitude can be minimised. Indeed, at the system set up, the observed phase value may be considered a random parameter, uniformly distributed between 0 and $2 \pi$. A first step consists of removing this random value by setting it to a predetermined value through

\section{Without Self-Tuning Network \\ -.-.-With Self-Tuning Network: Amplitude\&Phase Algorithm \\ With Self-Tuning Network: Gradient Based Algorithm - - With Self-Tuning Network: Only Phase Algorithm}
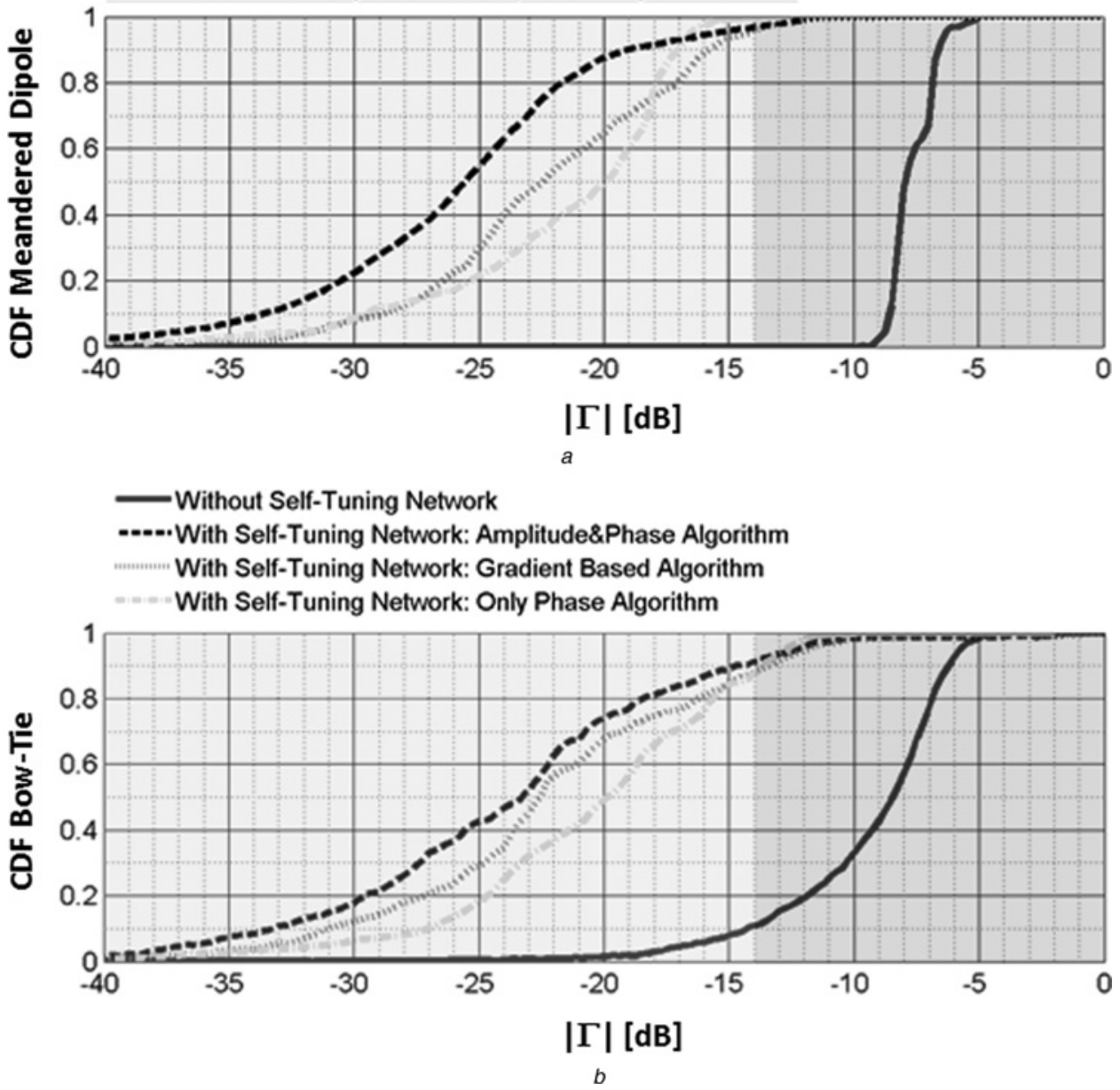

Fig. 6 Measured CDF of the reflection coefficient

$a \mathrm{CDF}$ for the meandered dipole when the antenna was worn by a volunteer $b$ CDF for the bowtie antenna when the antenna was worn by a volunteer $c$ CDF for the meandered dipole in proximity of water

$d \mathrm{CDF}$ for the bowtie antenna in proximity of water 

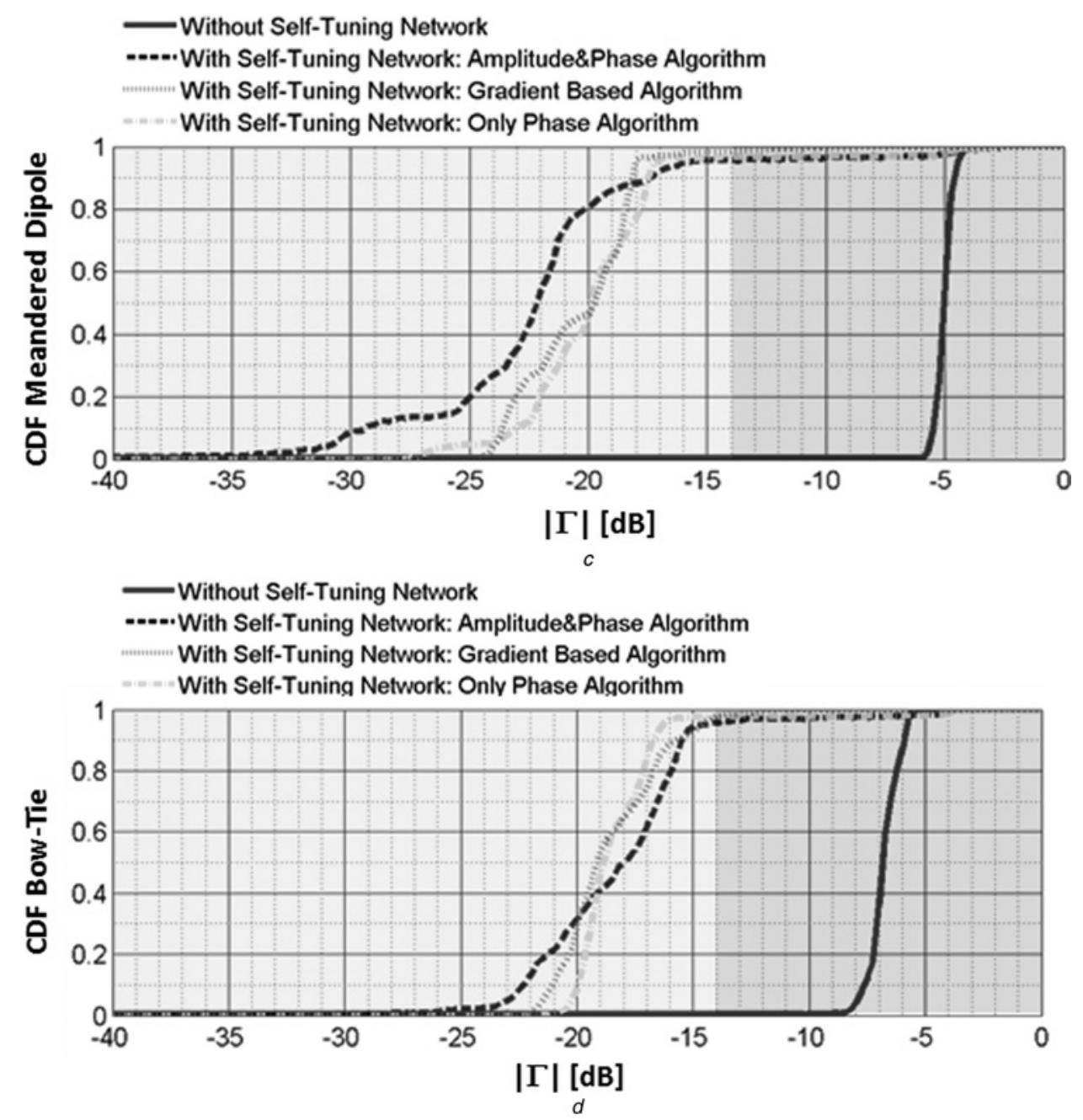

Fig. 6 Continued

proper modification of the two variable elements. Next, the two variable varicap diodes are varied to reach the centre of the Smith chart. The two capacitors are adapted in a similar way as for the default algorithm, summarised in Fig. 5. The first step requires modifying $C_{1}$ and $C_{2}$ to reach the phase-lock region. Shunt capacitor $C_{1}$ is so modified to select the proper conductance circles in which the reflection coefficient could move by varying the shunt capacitor $C_{2}$. This region is defined as the sector of the Smith chart between -45 and $45^{\circ}$. A phase lock region located in the left half-plane has been discarded, because we cannot define a unique set of actions on capacitor $C_{1}$ to approach the centre of the Smith chart from this area. Specifically, the value $C_{1}$ should be reduced if the reflection coefficient is located outside of the unitary value circle, and it should be increased if the reflection coefficient is located inside the unitary value circle. From within the phase-lock region, we can reach the centre of the Smith Chart, by first reducing $C_{1}$, while adapting $C_{2}$ to maintain phase lock.

The lack of the magnitude of the reflection coefficient as input data for the algorithm has an impact also on the definition of a stopping criterion. When fixing the angular sector for the phase lock on the Smith chart, we must consider that, when approaching the centre in the second phase of the algorithm, the closer the actual reflection coefficient comes to the centre of the Smith chart, the easier it is to exit from the above-defined angular sector. Therefore, nearest neighbour check is performed to evaluate the proximity to the centre of the Smith chart. The width of the sector angle and the step variation on the capacitance represent a trade-off between convergence speed and precision for the impedance matching.

\section{$4 \quad$ Test and measurement results}

To validate and test the realised prototype, a meandered dipole antenna and a bowtie antenna [6] have been connected to the user terminal during the measurements. The main characteristics observed during the validation of the system prototype are the insertion loss of the impedance matching network and the input impedance of the transmitting element. On the one hand, the loss analysis is important because large losses cause a significant performance degradation of the overall system, limiting the benefits of the self-tuning network. On the other hand, the reduction of the reflected power at the antenna input improves the probability of successful transmission by the distress beacon during an emergency situation.

During the tests, the Cospas-Sarsat system prototype was worn by a volunteer who performed a series of random movements. Improvements in terms of reflection coefficient are evaluated by comparing the measured antenna reflection coefficient with and without the self-tuning network for all three developed algorithms. Six hundred samples of the reflection coefficient were collected for $5 \mathrm{~min}$. The cumulative density functions (CDFs) of the collected data are shown in Fig. 6, for different cases. The improvements introduced by the self-tuning network are visible in terms of a reduced mean value of the reflection coefficient, decreasing from $-8.4 \mathrm{~dB}$ (in case of the meandered dipole) and $-9.5 \mathrm{~dB}$ (for the bowtie antenna) to below $-20 \mathrm{~dB}$ for all the algorithms and for both antennas. Fig. 6 clearly shows that, by applying self-tuning, the reflection coefficient now meets the specification of being below the reference value of $-14 \mathrm{~dB}$ in almost all circumstances. Indeed, when the meandered antenna is worn by a volunteer, the matching requirement is never satisfied without matching network, 


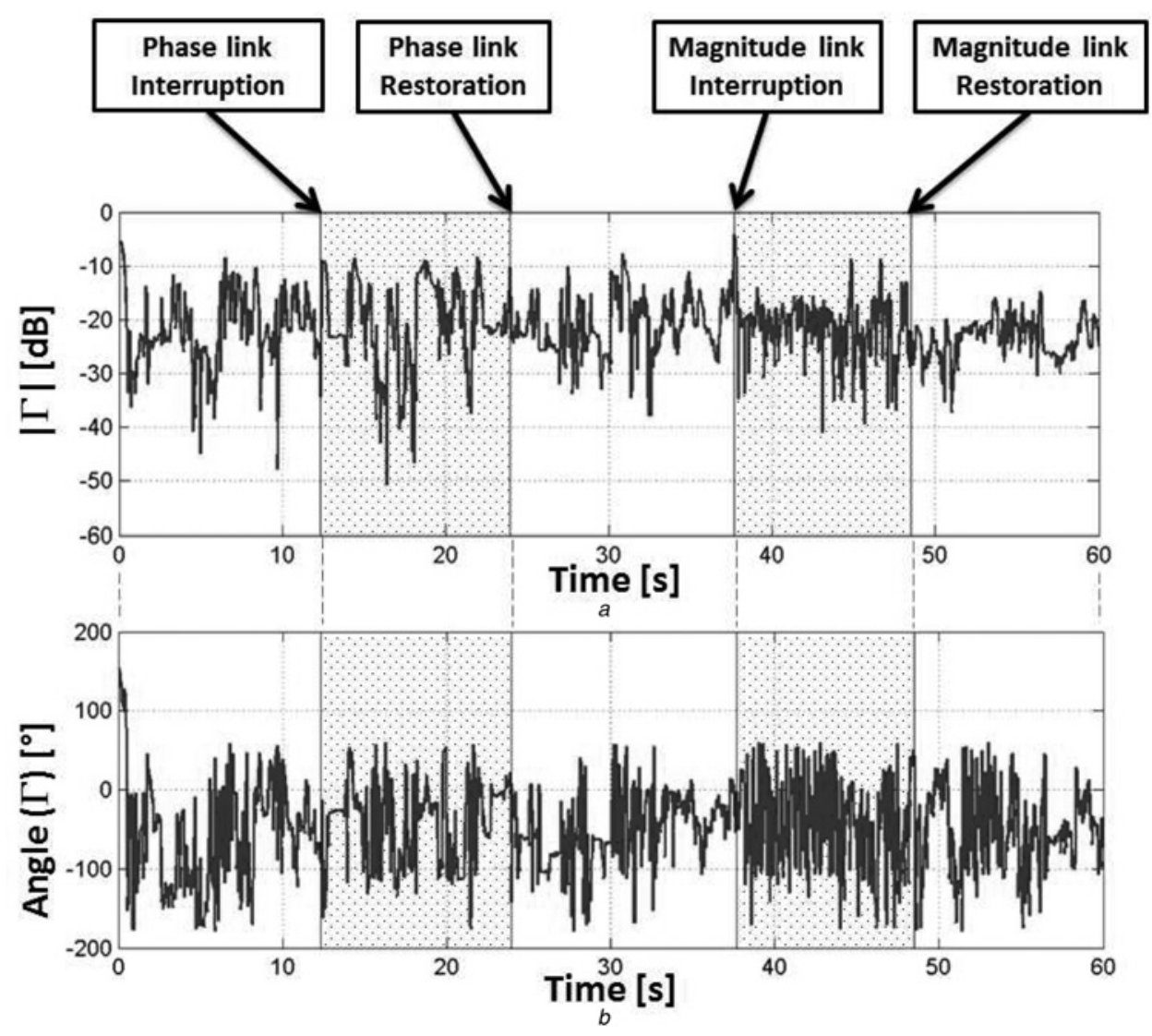

Fig. 7 Measurement of the reflection coefficient when the antenna was worn by a volunteer during a failure emulation $a$ Absolute value of the reflection coefficient $b$ Phase of the reflection coefficient

whereas with tuning circuit the requirements are fulfilled during $95 \%$ of the time. For the bowtie antenna worn by a person, we obtain a better than $-14 \mathrm{~dB}$ reflection coefficient only during $11 \%$ of the time, rising up to $87 \%$ after tuning by any of the three algorithms. Similar results were obtained when the antenna is deployed in the proximity of water. Starting from a situation in which the $-14 \mathrm{~dB}$ criterion was not fulfilled at all, the impedance matching network reduces the reflection coefficient to below the $-14 \mathrm{~dB}$ threshold in more than $95 \%$ of the time, for both antennas and with all three deployed algorithms.

To validate the resilience of the realised software architecture, several tests were conducted while the input data (amplitude and phase of the reflection coefficient) were dynamically made available or unavailable to the microcontroller by a software enabling system. With these tests, the ability of the software architecture to switch between default and backups algorithms has been proved.

Different combinations of available input data have been tried in different tests. In Fig. 7, an example of collected data over a time span of $60 \mathrm{~s}$ is shown. At the system start-up, both absolute value and phase of the reflection coefficient were available as input data to the microcontroller. During this time frame, the default algorithm was applied to achieve the impedance matching. Later on, the phase link was interrupted for $12 \mathrm{~s}$ before being restored, causing the microcontroller to switch from the default algorithm to backup algorithm \#1, and back. The switch between the default algorithm and the backup algorithm \#2 was tested in the last part, by interrupting the magnitude link information. The latter was interrupted for $12 \mathrm{~s}$ before being restored. With these actions, all the possible paths shown in the flowchart in Fig. $4 a$ have been explored.

With this time domain analysis, the reaction of the software architecture in response to changes in the physical circuit is demonstrated. Moreover, a deeper time domain analysis permits to appreciate and to better understand the working principles of the different algorithms.
Indeed, by enlarging the time scale at the system start-up and observing the first output data of the reflection coefficient on the Smith chart, it is possible to appreciate the adaptation process of the default algorithm (Figs. $8 a$ and $b$ ). In this case, at the system start-up the reflection coefficient is located in region \#2. By adapting both variable capacitors, we first lead the reflection coefficient to the unitary admittance circle (where capacitor $C_{1}$ is responsible for compensating for the real part of the admittance), which is then directed to the centre of the Smith chart (by varying capacitor $C_{2}$ to compensate for the imaginary part of the admittance).

We now discuss backup algorithm \#2, which only uses the phase of the reflection coefficient for the impedance matching. By concentrating on the measurements performed around time instant $38 \mathrm{~s}$, we can observe the two initial steps described in Section 3 . The developed algorithm requires a preliminary phase, where the phase of the reflection coefficient is led to a phase-lock value, followed by the main phase, where the reflection coefficient is moved towards the centre along the lock angle. This characteristic can be observed in Figs. $8 c$ and $d$. In the dashed part, the microcontroller is leading the phase towards the lock value, whereas in the dotted part the reflection coefficient is lead towards the centre of the Smith chart along the lock-value phase.

All algorithms exhibit a convergence time that basically depends on the initial position of the reflection coefficient in the Smith chart. Yet, algorithms and antennas we tested, converged to a reflection coefficient below $-10 \mathrm{~dB}$ within a time frame of $300 \mathrm{~ms}$. After matching is obtained, the system is able to maintain the reflection coefficient value below $-10 \mathrm{~dB}$ during more than $99 \%$ of the time within a 5 min' time span, when the antenna was worn by a volunteer performing random movements, as depicted in Fig. 7. Also in those cases in which the reflection coefficient increases to values above $-10 \mathrm{~dB}$, due to the wearer's movements, all algorithms adapt and achieve matching again in a few $100 \mathrm{~ms}$. The measured convergence time and response time is considered 

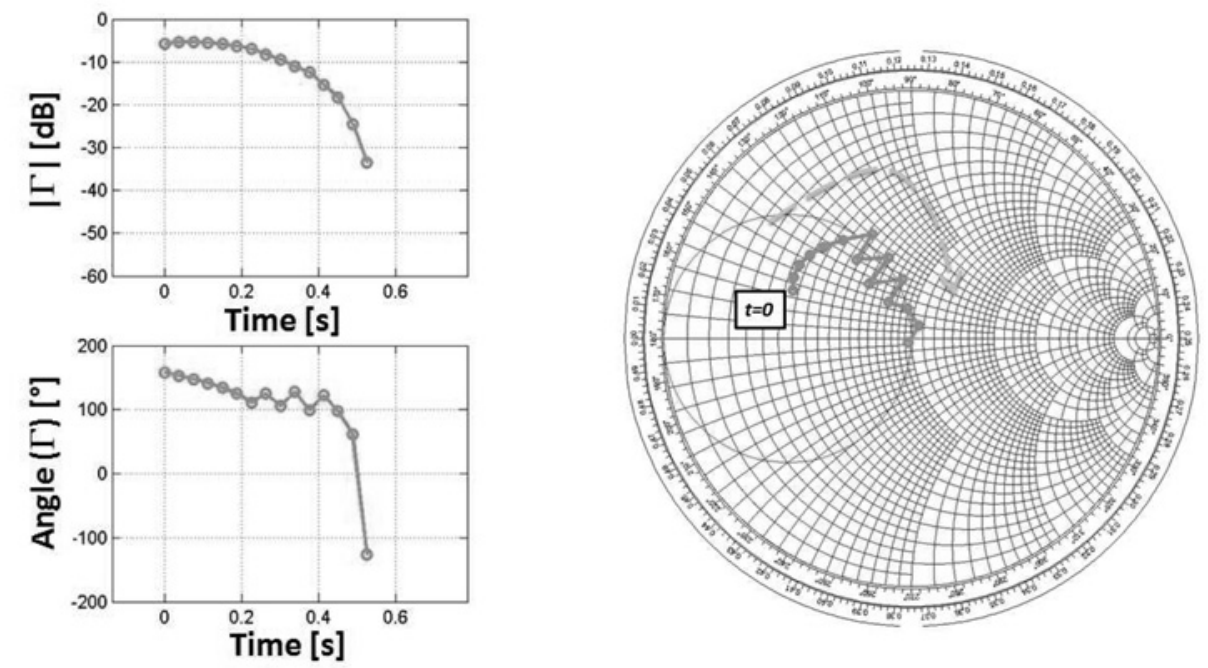

a

$b$
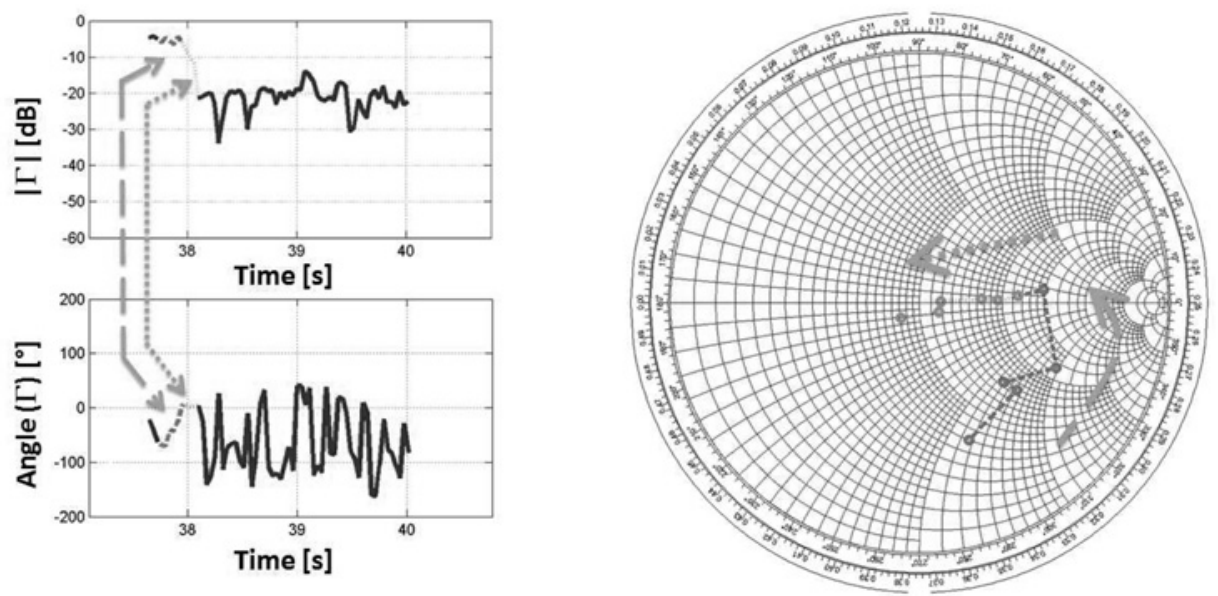

Fig. 8 Detail of the measured reflection coefficient during the automated tuning procedure

a Absolute value and angle of the measured reflection coefficient over the time using the default algorithm

$b$ Collected reflection coefficient into the Smith chart using the default algorithm

$c$ Absolute value and angle of the collected reflection coefficient over the time using the backup algorithm \#2 (only phase information available to the microcontroller)

$d$ Collected reflection coefficient into the Smith chart using the backup algorithm \#2 (only phase information available to the microcontroller)

adequate for being applied to a Cospas-Sarsat system when a half-second burst is transmitted approximately every $50 \mathrm{~s}$. On the basis of the collected results, the first burst could be transmitted after half a second from the system start-up, in order to ensure that the system achieves matching after being initiated. For the following bursts, the probability of having a transmission during an antenna mismatch condition is lower than $1 \%$. To enlarge the life span of the system even more, the self-tuning network could be switched off and turned on only a few seconds before signal burst transmission.

The measured insertion loss is around $1.9 \mathrm{~dB}$. This value is mainly attributed to the applied wideband directional couplers, which have a total insertion loss of $1.8 \mathrm{~dB}$. The use of dedicated narrowband direction couplers could reduce the total insertion loss of the self-tuning circuit, thereby improving the overall circuit efficiency.

\section{Conclusion}

A self-tuning network for a wearable Cospas-Sarsat transmitting system has been designed, built and tested. The realised low-cost self-tuning network is able to estimate the actual reflection coefficient and to tune a dedicated impedance network accordingly by a dedicated robust software architecture. The systems increase the probability of correct distress beacon signal transmission in harsh operating conditions by ensuring that the Cospas-Sarsat requirements remain satisfied at all time. The tuning network relies on varicap diodes, whose capacities are modified in real-time based on the antenna's measured reflection coefficient. The matching process is executed by adaptively controlling the tuning network parameters. Three different algorithms were implemented that rely on different feedback information from the gain/phase detector. Although all three algorithms can individually perform the impedance matching, the realised default/backup software architecture improves the reliability of the wearable device when deployed in an emergency situation. Moreover, the generic software architecture implementing the backup algorithms described in this paper can be applied in conjunction with many low-cost off-the-shelf components that do not provide both the amplitude and phase of the reflection coefficient, but only one of the mentioned quantities. The system may be connected to different wearable Cospas-Sarsat antennas, making it suitable for integration into a commercial life-jacket. It has been experimentally shown that detuning of antenna's resonance frequency due to the antennabody interaction and the wearer's natural movements can be effectively compensated, limiting the antenna's reflection coefficient to below $-14 \mathrm{~dB}$, as requested by the Cospas-Sarsat specification. Future work will focus on the realisation of an even more compact low-cost version of the proposed system. 


\section{References}

1 'International Cospas-Sarsat Programme' http://www.cospas-sarsat.int/, accessed 17 August 2015

2 King, J.V.: 'Cospas-Sarsat: an international satellite system for search and rescue', Space Commun., 2002, 18, (3-4), pp. 139-150

3 Nepa, P., Manara, G.: 'On the stochastic characterization of wearable antennas' PIERS 2013 (Progress in Electromagnetics Research Symp.), Stockholm, Sweden, 2013, p.

4 Hall, P.S., Yang Hao, Nechayev, Y.V.I., et al.: 'Antennas and propagation for on-body communication systems', IEEE Antennas Propag. Mag., 2007, 49, (3), pp. 41-58

5 Nepa, P., Rogier, H.: 'Wearable antennas for off-body radio links at VHF and UHF bands (below $1 \mathrm{GHz}$ ): challenges, state-of-the-art and future trends', IEEE Antennas Propag. Mag., 2015, 57, (5)

6 Serra, A., Nepa, P., Manara, G.: 'A wearable two-antenna system on a life jacket for Cospas-Sarsat personal locator beacons', IEEE Trans. Antennas Propag., 2012, 60, (2), pp. 1035-1042

7 Specification for Cospas-Sarsat $406 \mathrm{MHz}$ Distress Beacons, https://www.cospassarsat.int/images/stories/SystemDocs/Current/CS-T-001-Oct2014.pdf accessed 17 August 2015

8 Lilja, J., Pynttari, V., Kaija, T., et al.: 'Body-worn antennas making a splash: lifejacket-integrated antennas for global search and rescue satellite system', IEEE Antennas Propag. Mag., 2013, 55, (2), pp. 324-341

9 Fu, J.S., Zhu, X.A., Phillips, J.D., et al. 'A ferroelectric-based impedance tuner for adaptive matching applications', IEEE MTT-S Int. Microwave Symp. Digest, 2008, 2008, pp. 955-958

10 Sun, Y., Moritz, J., Zhu, X.: 'Adaptive impedance matching and antenna tuning for green software-defined and cognitive radio', IEEE 54th Int. Midwest Symp. on Circuits and Systems (MWSCAS), 2011, August 2011, pp. 1-4

11 Firrao, E.L., Annema, A.J., Nauta, B.: 'An automatic antenna tuning system using only RF signal amplitudes', IEEE Trans. Circuits Syst. II, Express Briefs, 2008, 55, (9), pp. 833-837

12 Nabki, F., Deslandes, D.: 'An agile matching network using phase detection for antenna tuning', IEEE 20th Int. Conf. Electronics, Circuits, and Systems (ICECS), 2013

13 Gu, Q., De Luis, J.R., Morris, A.S., et al.: 'An analytical algorithm for pi-network impedance tuners', IEEE Trans. Circuits Syst. I, Regul. Pap., 2011, 58, (12), pp. 2894-2905

14 Gu, Q., Morris, A.S.: 'A new method for matching network adaptive control', IEEE Trans. Microw. Theory Tech., 2013, 61, (1), pp. 587-595
15 Song, H., Oh, S.H., Aberle, J.T., et al.: 'Automatic antenna tuning unit for software-defined and cognitive radio', IEEE Antennas and Propagation Society Int. Symp., 2007, June 2007, pp. 85-88

16 De Mingo, J., Valdovinos, A., Crespo, A., et al.: 'An RF electronically controlled impedance tuning network design and its application to an antenna input impedance automatic matching system', IEEE Trans. Microw. Theory Tech., 2004, 52, (2), pp. 489-497

17 Tan, Y., Sun, Y., Lauder, D.: 'Automatic impedance matching and antenna tuning using quantum genetic algorithms for wireless and mobile communications', IET Microw. Antennas Propag., 2013, 7, (8), pp. 693-700

18 Sánchez, C., de Mingo, J., Garcia, P., et al.: 'Application of an impedance tuning network for mobile DVB-H terminals', IEEE 70th Vehicular Technology Conf. Fall (VTC 2009-Fall), 2009, September 2009, pp. 1-5

19 Coleman, C.M., Rothwell, E.J., Ross, J.E.: 'Investigation of simulated annealing, ant-colony optimization, and genetic algorithms for self-structuring antennas', IEEE Trans. Antennas Propag., 2004, 52, (4), pp. 1007-1014

20 Chen, Y., Manteuffel, D.: 'Miniaturizing of a distributed MEMS impedance matching network'. Int. Workshop on Antenna Technology (iWAT), 2013, March 2013, pp. 303-306

21 Sharma, A.K., Gupta, N.: 'Impedance matching for RF-MEMS based microstrip patch antenna'. 11th Int. Conf. on Electrical Engineering/Electronics, Computer, Telecommunications and Information Technology (ECTI-CON), 2014, May 2014, pp. 1-4

22 Huang, L., Russer, P.: 'Electrically tunable antenna design procedure for mobile applications', IEEE Trans. Microw. Theory Tech., 2008, 56, (12), pp. 2789-2797

23 Sharma, V., Pathak, N.P. 'Continuously tunable concurrent dual-frequency impedance matching network', 7th IEEE Int. Conf. on Industrial and Information Systems (ICIIS), 2012, August 2012, pp. 1-3

24 Chen, Y., Martens, R., Valkonen, R., et al: 'A varactor-based tunable matching network for a non-resonant mobile terminal antenna', 8th European Conf. on Antennas and Propagation (EuCAP), 2014, April 2014, pp. 1877-1881

25 Jang, H., Son, W., Oh, K., et al.: 'High-speed real-time hand effect tuning algorithm in hand-held terminal', IEEE MTT-S Int. Microwave Workshop Series on Intelligent Radio for Future Personal Terminals (IMWS-IRFPT), 2011, August 2011, pp. 1-2

26 'Varicap' diode datasheet, http://www.onsemi.com/pub_link/Collateral/ MMBV105GLT1-D.PDF, accessed 17 August 2015

27 Directional Coupler datasheet, http://cdn.macom.com//datasheets/MACP-011013. pdf, accessed 17August 2015 